\title{
AN ISVD-BASED EUCLIDIAN STRUCTURE FROM MOTION FOR SMARTPHONES
}

\author{
A. Masiero ${ }^{\text {a, } *}$, A. Guarnieri ${ }^{\mathrm{a}}$, A. Vettore ${ }^{\mathrm{a}}$, F. Pirotti $^{\mathrm{a}}$ \\ ${ }^{\text {a }}$ Interdepartmental Research Center of Geomatics (CIRGEO), University of Padova, \\ Viale dellUniversit 16, Legnaro (PD) 35020, Italy - \\ masiero@dei.unipd.it \\ (alberto.guarnieri, antonio.vettore, francesco.pirotti)@unipd.it
}

Commission V WG 4

KEY WORDS: Reconstruction, Georeferencing, Calibration, Camera, Real-time, Photogrammetry

\begin{abstract}
:
The development of Mobile Mapping systems over the last decades allowed to quickly collect georeferenced spatial measurements by means of sensors mounted on mobile vehicles. Despite the large number of applications that can potentially take advantage of such systems, because of their cost their use is currently typically limited to certain specialized organizations, companies, and Universities. However, the recent worldwide diffusion of powerful mobile devices typically embedded with GPS, Inertial Navigation System (INS), and imaging sensors is enabling the development of small and compact mobile mapping systems.

More specifically, this paper considers the development of a 3D reconstruction system based on photogrammetry methods for smartphones (or other similar mobile devices). The limited computational resources available in such systems and the users' request for real time reconstructions impose very stringent requirements on the computational burden of the 3D reconstruction procedure.

This work takes advantage of certain recently developed mathematical tools (incremental singular value decomposition) and of photogrammetry techniques (structure from motion, Tomasi-Kanade factorization) to access very computationally efficient Euclidian 3D reconstruction of the scene.

Furthermore, thanks to the presence of instrumentation for localization embedded in the device, the obtained 3D reconstruction can be properly georeferenced.
\end{abstract}

\section{INTRODUCTION}

The developments of photogrammetry during the last decades allowed to obtain high resolution 3D models of the reality from (quite low cost) camera measurements by means of well known and typically quite computational demanding signal processing procedures (data association and structure from motion methods). Despite some issues (e.g. related to the illumination conditions of the scene) may occur, the quality of the 3D reconstructions obtained by means of photogrammetry methods are typically comparable with those obtained by means of other, more expensive, sensors.

Nowadays, the world-wide capillary diffusion of low cost mobile cameras and smartphones and the users' demand for 3D augmented reality systems, possibly quickly obtained by using mobile devices, are motivating the development of computationally efficient $3 \mathrm{D}$ reconstruction methods.

This work deals with the development of a 3D reconstruction system based on measurements from an uncalibrated camera, typically embedded in a (usually low cost) smartphone. In addition, the goal is to obtain a georeferenced 3D model by exploiting the measurements of a proper navigation system based on both GPS and the inertial navigation system of the smartphone.

The main challenge in the development of such 3D reconstruction systems is the reduction of the computational efforts requested by classical methods for 3D reconstruction from uncalibrated cameras. Recently, some methods have been proposed in the literature to reduce such computational effort: most of them are based on the optimization of bundle adjustment methods, e.g. the use

${ }^{*}$ Corresponding author. of Preconditioned Conjugate Gradients to speed up the bundle adjustment optimization (Agarwal et al., 2010, Byröd and Aström, 2010).

Differently from such methods, this work takes advantage of the Incremental Singular Value Decomposition (ISVD (Brand, 2002)) to obtain a fast factorization of the measurement matrix (Tomasi and Kanade's factorization (Tomasi and Kanade, 1992)). This procedure, which has been recently proposed by Kennedy et al. (Kennedy et al., 2013), allows to quickly obtain a projective reconstruction of the scene.

This paper proposes the integration of the above factorization algorithm with the information provided by the navigation system embedded in the device in order to obtain a fast and effective georeferenced reconstruction of the Euclidian 3D structure of the scene: thanks to the use of computationally efficient methods, the overall reconstruction procedure can be executed in real time on standard mobile devices, e.g. smartphones.

Since the proposed procedure allows to obtain georeferenced 3D reconstructions, the outcomes of this method can be used both as reconstructions for an imaging system, and as feedback information for the navigation system in order to improve its localization.

\section{SYSTEM DESCRIPTION}

This work assumes the use of a (typically low cost) mobile device (e.g. a smartphone). Such device has to be provided of an imaging sensor (i.e. a camera), and of a navigation system.

The proposed system exploits the embedded camera to estimate a 3D reconstruction of the scene by means of a Structure from 
Motion (SfM) approach (Hartley and Zisserman, 2003, Ma et al., 2003). The device is moved on several locations, where the user takes shots of the scene by means of the camera embedded in the device. 3D reconstruction is accessed by relating with each others features in shots taken from different point of views.

Nowadays, most of the cameras mounted on standard smarthones have a resolution of several Mega-pixels, usually sufficient to provide accurate $3 \mathrm{D}$ reconstructions. Hence, the proposed approach does not impose specific requirements on the camera characteristics.

However, SfM methods allow to reconstruct the scene up to a scale factor (Hartley and Zisserman, 2003, Fusiello, 2000, Chiuso et al., 2000), then information on the device position provided by the navigation system has to be exploited in order to estimate the unknown scale factor and the georeferenced spatial position. Outdoors the GPS system can be sufficient to provide estimations of the device position (Piras et al., 2010), however indoors the GPS signal is usually not available (or not reliable). In order to allow position estimation in indoors conditions, the considered device is assumed to be provided of an INS based on embedded sensors as well: in particular, the considered smartphone is assumed to be provided of a 3-axis accelerometer and of a 3-axis magnetometer. Simultaneous measurements by such instruments allow the estimation of both the movements of the mobile device, and the attitude of the device during the camera shot.

Information on the position and the attitude of the device can be exploited in the SfM algorithms as initial conditions for iterative optimization methods of the parameters, in order to speed up the convergence. Furthermore, they can be used to reduce the burden of the image processing step by allowing a smart selection of images to be analyzed in order to find matched features.

Outdoor localization based on the use of the GPS signal can be considered as a quite standard procedure. Instead, several algorithms have been recently proposed in the literature in order to access indoor position estimation based on the combined use of different sensors (Azizyan et al., 2009, Bahl and Padmanabhan, 2000, Cenedese et al., 2010, Foxlin, 2005, El-Sheimy et al., 2006, Guarnieri et al., 2013, Lukianto and Sternberg, 2011, Masiero et al., 2013, Ruiz et al., 2012, Youssef and Agrawala, 2005, Wang et al., 2012, Widyawan et al., 2012). In the procedure described in the following section it is assumed that estimations of the device position and attitude are available. In our current implementation the positioning system is as in (Masiero et al., 2013), however different choices can be considered without affecting the effectiveness of the procedure.

Our current implementation of the system is on a low cost mobile phone, shown in Fig. 1. The developed application shall be executed on most the Android phones (with the above specifications on the embedded sensors).

\section{ITERATIVE RECONSTRUCTION}

The reconstruction procedure is assumed to process data iteratively: when a new image shoot is available a new iteration of the reconstruction procedure starts. Each iteration of the reconstruction process can be decomposed in the following steps (Fig. 2) that will be detailed in the following subsections: feature extraction and matching, projective reconstruction and Euclidian promotion.

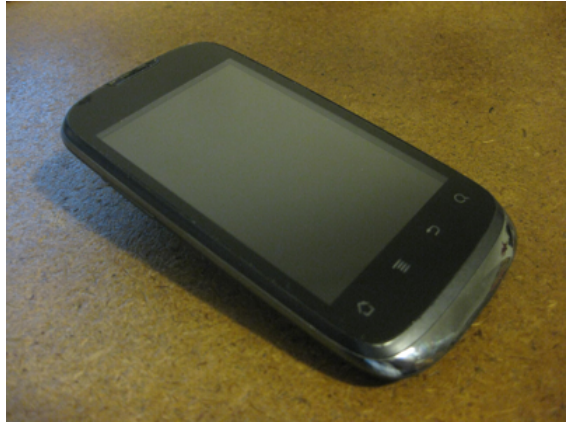

Figure 1: Smartphone used to test the reconstruction system: Huawei U8650 Sonic.

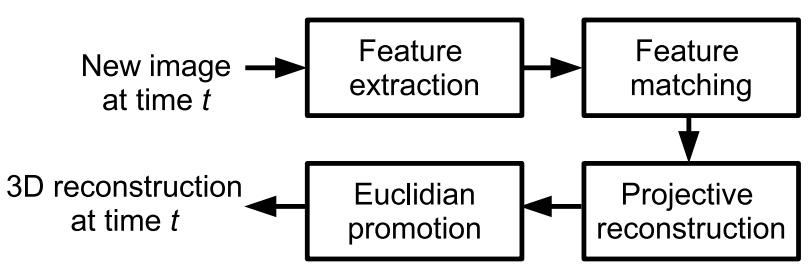

Figure 2: Reconstruction procedure scheme.

\subsection{Feature extraction and matching}

The considered procedure relies on a feature based approach for $3 \mathrm{D}$ reconstruction: the geometry of the scene is reconstructed by analyzing the position of features from different point of views. In order to properly estimate the geometry of the scene the same spatial point has to be recognized and matched in the images where it is visible. Hence the use of a proper feature extraction and matching technique is of fundamental importance in order to ensure the effectiveness of the reconstruction procedure.

Feature extraction and matching here is decomposed in a two step procedure: first, feature extraction has been implemented by means of the Affine Scale-Invariant Feature Transform (ASIFT) (Morel and Yu, 2009): ASIFT provides a method for reliable feature matching among different images. Feature matching based on ASIFT is based on the appearance of the 2D images: since images are taken from different point of views the same feature can undergo certain appearance changes, the goal of the ASIFT method is that of extracting features invariant to such deformations, that are locally modeled as affine transforms.

Despite the ASIFT method is reliable to compensate for local changes in feature appearance, unfortunately mismatches are unavoidable in certain cases, in particular when dealing with repetitive structures, e.g. in human buildings. Then, similarly to (Snavely et al., 2008), in order to make feature matching more reliable in such critical conditions, a matching step based on the reconstructed geometry has been implemented as well. After matching points between two images through the ASIFT descriptors, the system use a RANSAC approach (Fischler and Bolles, 1981) to make a robust feature selection: the system iteratively selects 8 matched points, and, based on them, computes a candidate geometry transformation between the two images (i.e. the fundamental matrix by using the eight-point algorithm as in (Longuet-Higgins, 1981, Hartley, 1997, Hartley and Zisserman, 2003)). Then, at each iteration the number of features that are properly matched by means of the estimated fundamental matrix $F$ is counted: after the last iteration of the RANSAC algorithm, the matrix $F$ with the highest number of properly matched features is selected, and the corresponding properly matched features are considered as properly associated in the following steps of the procedure (Fig. 3). 
In principle, features detected in a new image should be matched with the features of all the other frames. However, as the number of images increases such procedure becomes computationally demanding. Several techniques based on different similarity measures have been previously proposed in the literature to match features only for "highly correlated" images (Furukawa et al., 2010, Goesele et al., 2007, Masiero and Cenedese, 2013). The rationale among such image selection methods is that images to be compared should be reasonably similar to have a large number of common features, however they should also have different point of views (e.g. a large baseline) to ensure a good reconstruction of the feature spatial positions. In this paper we take advantage of the information on the device position and orientation to select the small set of images to compare with the current one for feature matching: the selection is done using considerations on the expected reconstruction error modeled similarly to (Masiero and Cenedese, 2013).
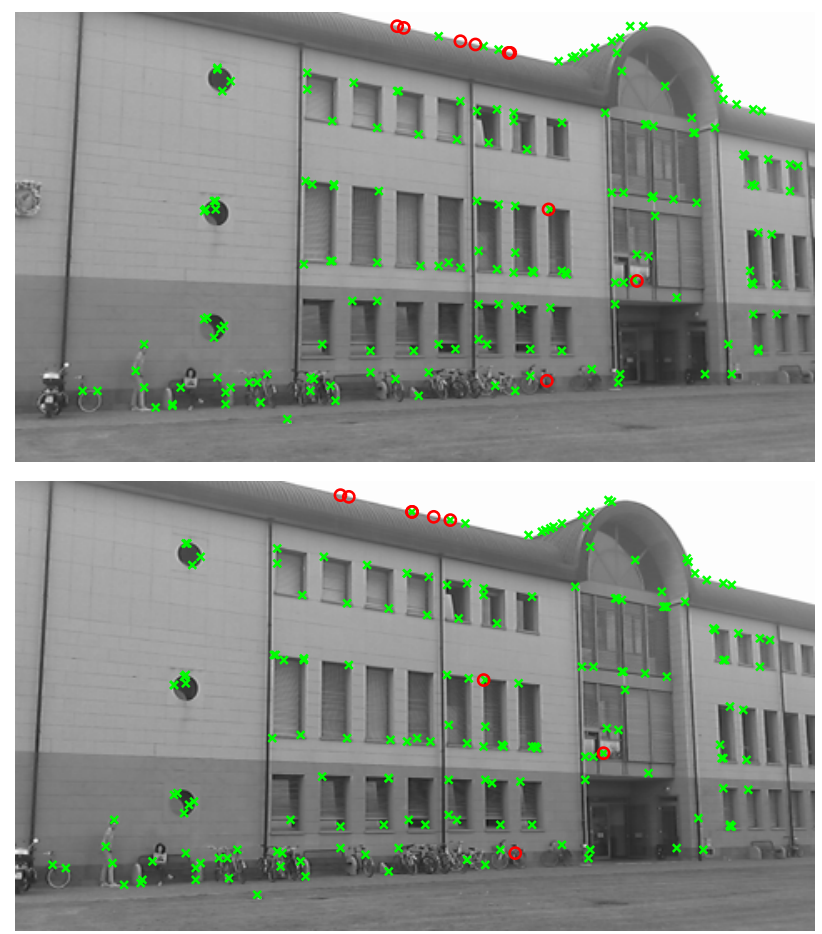

Figure 3: Feature extraction: example of features matched by means of the ASIFT method in two images. Features considered as properly matched after the execution of the RANSAC algorithm are shown as green circles. Mismatches are shown as red circles.

\subsection{Projective reconstruction based on ISVD}

This subsection presents a projective reconstruction procedure based on the ISVD (Brand, 2002). The approach is similar to that in (Kennedy et al., 2013), however while the work in (Kennedy et al., 2013) was limited to affine cameras, here projective cameras are considered. Furthermore, the projective reconstruction obtained in this subsection will be generalized to an Euclidian reconstruction and georeferenced in the next subsection.

Since the camera embedded in the mobile device is modeled as a projective camera, then the measurement $m_{i j}$ of feature $j$ on the image plane of camera view $i$ can be related with its corresponding $3 \mathrm{D}$ point $M_{j}$ as follows:

$$
m_{i j} \xi_{i j}=P_{i} M_{j}
$$

where $P_{i}$ is the projective matrix of camera view $i$ (taking into account also of the camera focal length), $\xi_{i j}$ is the distance of the optical center of the camera in view $i$ from the orthogonal projection of $M_{j}$ on the line corresponding to the optical axis of camera in view $i . m_{i j}$ and $M_{j}$ are written by using homogeneous coordinate notation (Ma et al., 2003).

The above equation can be generalized for the case of $\boldsymbol{m}$ different views and $n$ feature points:

$$
\left[\begin{array}{ccc}
m_{11} \xi_{11} & \ldots & m_{1 n} \xi_{1 n} \\
\vdots & & \vdots \\
m_{\boldsymbol{m} 1} \xi_{11} & \ldots & m_{\boldsymbol{m} n} \xi_{1 n}
\end{array}\right]=\left[\begin{array}{c}
P_{1} \\
\vdots \\
P_{\boldsymbol{m}}
\end{array}\right]\left[M_{1} \ldots M_{n}\right]
$$

where all the features are assumed to be visible in the views. By construction, matrices $M$ and $P$ have rank $\leq 4$ (however when estimated by real data this condition is usually not exactly satisfied because of the presence of noise). (2) is also referred to as the Tomasi and Kanade's factorization (Tomasi and Kanade, 1992).

Let the measurement matrix $W$ be defined as the matrix on the left side of equation (2). Then, the rationale of (2) is that as long as the measurement matrix $W$ is formed (and the values of the $\xi_{i j}$ are known) then the values of the projective matrices associated to camera views and the positions of the feature points can be estimated by means of the factorization of $W$, e.g. by using the Singular Value Decomposition (SVD):

$$
W=U S V^{\top}, P=U S(:, 1: 4), M=V(:, 1: 4)^{\top},
$$

where we have used Matlab-like notation for the matrix indices. Notice that matrices $P$ and $M$ are estimated up to a nonsingular matrix $T$, i.e.

$$
W=P M=(P T)\left(T^{-1} M\right)
$$

Notice that the above equation justifies also the arbitrary definition of $P$ and $M$ in (3).

While being very intuitive, the estimation of camera projection matrices and point positions with (2) have some drawbacks:

- First, the values of $\left\{\xi_{i j}\right\}$ are usually not exactly known. Nevertheless, the information about camera positions and orientations provided by the navigation system provide us with a usually quite good initial estimate of such values: then, a more reliable estimation can be obtained by iterating the estimation step of $P$ and $M$ described above, and an estimation step of the $\left\{\xi_{i j}\right\}$ from the estimated $P$ and $M$.

- The computational complexity for computing the SVD of the $W$ matrix (with size $(3 \boldsymbol{m}) \times n$ ) is approximatively $O\left(n \boldsymbol{m}^{2}\right)$, where $n \geq(3 \boldsymbol{m})$. When dealing with a large number of camera views and of feature points such computational cost can become prohibitive for the considered real-time application.

- Finally, the computation of $P$ and $M$ as in (2) does not allow to deal with features visible only in a subset of the $\boldsymbol{m}$ available camera views. 
Nevertheless, an iterative approach can be considered in order to tackle the last two of the above drawbacks: when a new image is available a new iteration of the algorithm for the estimation of $P$ and $M$ is started. In order to reduce the computational complexity of such iteration, the new solution is computed as an update of the previous solution, e.g. the new iteration is initialized by means of the previously estimated projection matrices and reconstructed feature points. A brief review of the updating rule of the ISVD will be presented in the following, for a more detailed description the reader is referred to (Brand, 2002).

Let $W_{t}$ be the matrix decomposed after $t$ iterations of the algorithm (e.g. that formed by the measured features extracted by $t$ views), then:

$$
W_{t} \approx U_{t} S_{t} V_{t}^{\top}
$$

where $U_{t}$ and $V_{t}$ have $h$ columns (typically $4 \leq h \leq \operatorname{rank}\left(W_{t}\right)$ ). At the $(t+1)$-th iteration, let $W_{t+1}=\left[W_{t} w_{t+1}\right]$ where $w_{t+1}$ is a proper column vector. Then, the (approximate) factorization of $W_{t+1}$ can be obtained as follows:

$$
\begin{aligned}
W_{t+1}=\left[W_{t} \mid w_{t+1}\right] & \approx\left[U_{t} S_{t} V_{t} \mid w_{t+1}\right] \\
& \approx\left[U_{t} S_{t} V_{t} \mid U_{t} v_{t+1}+r_{t+1}\right]
\end{aligned}
$$

where $v_{t+1}=U_{t}^{\dagger} w_{t+1}$ (where $U_{t}^{\dagger}$ stands for the pseudo-inverse of $U_{t}, U_{t}^{\dagger}=U_{t}^{\top}$ for unitary $U_{t}$ ) and $r_{t+1}=w_{t+1}-U_{t} v_{t+1}$.

Rearranging the above equation it immediately follows that:

$$
W_{t+1} \approx\left[U_{t} \mid \frac{r_{t+1}}{\left\|r_{t+1}\right\|}\right]\left[\begin{array}{cc}
S_{t} & v_{t+1} \\
0 & \left\|r_{t+1}\right\|
\end{array}\right]\left[\begin{array}{cc}
V_{t} & 0 \\
0 & 1
\end{array}\right]^{\top}
$$

Let the matrix $\left[\begin{array}{cc}S_{t} & v_{t+1} \\ 0 & \left\|r_{t+1}\right\|\end{array}\right]$ be factorized by the SVD algorithm as follows:

$$
\left[\begin{array}{cc}
S_{t} & v_{t+1} \\
0 & \left\|r_{t+1}\right\|
\end{array}\right] \approx \tilde{U}_{t} \tilde{S}_{t} \tilde{V}_{t}^{\top}
$$

where $\tilde{S}_{t}$ is an $h \times h$ matrix. Then, the factorization of $W_{t+1}$ can be updated by taking:

$$
\begin{aligned}
U_{t+1} & =\left[U_{t} \mid \frac{r_{t+1}}{\left\|r_{t+1}\right\|}\right] \tilde{U}_{t} \\
S_{t+1} & =\tilde{S}_{t} \\
V_{t+1} & =\left[\begin{array}{cc}
V_{t} & 0 \\
0 & 1
\end{array}\right] \tilde{V}_{t}
\end{aligned}
$$

Notice that the above considerations can be easily extended to the case where $W_{t+1}$ is formed by adding a row to $W_{t}$.

The procedure described above allow to conveniently update the SVD factorization (and consequently the factorization of (2) in our case) in $O\left(n h^{2}\right)$ (for $\boldsymbol{m}<n$ and $h$ small with respect to both $n$ and $\boldsymbol{m}$ ). Since in the case of interest $h \ll \boldsymbol{m}$, then the ISVD allow to obtain a great speed up with respect to the direct use of the SVD.
Accordingly with the definition of the $W$ matrix, adding a column or a row to $W_{t}$ corresponds to add a new feature point or a new camera view, respectively. Hence, the use of the ISVD allows to reduce the computational effort needed to recompute the estimation of $P$ and $M$ when a new camera view or a new feature point are considered.

In order to deal with a feature point not available in all the camera views, when a new measurement of it is available one can select the part of the $W$ matrix corresponding to the views where such point is visible, and update such part with a procedure similar to that presented above (slightly adapted to take into account of the different conditions of use).

The procedure described above provides an efficient iterative factorization method of the measurement matrix $W$ that leads to a projective reconstruction of the scene.

\subsection{Euclidian promotion and georeferencing the system}

Accordingly to (4), the projective reconstruction of the scene estimated as in the previous subsection differs from an Euclidian reconstruction for a nonsingular matrix $T$. Furthermore, the final goal of our system is that of obtaining a georeferenced reconstruction, that differs from an Euclidian reconstruction for a translation, a rotation and a scale factor.

Two alternative approaches can be considered in order to compute a georeferenced reconstruction from the projective one.

In the first option, a two step procedure can be considered: first, estimate the Euclidian reconstruction (Euclidian promotion), and then use the estimated device positions to estimate the proper scale, translation and rotation that leads to the georeferenced reconstruction.

Several procedures have been proposed in the literature to tackle the Euclidian promotion problem (Hartley and Zisserman, 2003, Fusiello, 2000). For instance, the approach based on Kruppa's constraints described in (Heyden and Aström, 1996) can be adopted. Without loss of generality (Heyden and Aström, 1996, Fusiello, 2000), $T$ can be assumed to be as follows:

$$
T=\left[\begin{array}{cc}
K & 0 \\
r^{\top} & 1
\end{array}\right]
$$

where $K$ is the intrinsic parameter matrix (Ma et al., 2003), and $r$ is a $3 \times 1$ vector. Then, the estimates $\left\{\hat{P}_{i}\right\}_{i=1, \ldots, \boldsymbol{m}}$ of the camera projective matrices obtained from the projective reconstruction have to satisfy the following constraints

$$
\hat{P}_{i}\left[\begin{array}{cc}
K K^{\top} & K r \\
r^{\top} K^{\top} & r^{\top} r
\end{array}\right] \hat{P}_{i}^{\top}=\lambda_{i}^{2} K K^{\top}
$$

where $i=1, \ldots, \boldsymbol{m}$, and $\left\{\lambda_{i}\right\}$ are scale factors. As long as a sufficiently large number of camera views are available, and camera measurements are not ideal (i.e. corrupted by noise), then the above equation typically do not have an exact solution. Then, matrix $T$ is usually estimated by minimizing the sum of the squared differences between the left and right sides in (14) for $i=1, \ldots, \boldsymbol{m}$. Such solution is usually computed by means of iterative optimization methods (e.g. Gauss-Newton algorithm).

After the estimation of $T$, the transform between the considered Euclidian coordinate system and the georeferenced coordinate system has to be estimated. 
Since the goal of the procedure is to compute a georeferenced reconstruction, here an alternative approach can is considered, i.e. the direct estimation of the transform matrix $T_{g}$ from the coordinate system of the projective reconstruction to that of the georeferenced reconstruction.

Let $x_{i}$ be the (georeferenced) position of the device during the $i$-th camera acquisition, estimated by the navigation system. The estimate $x_{i}$ of the device position is assumed to be affected by a zero-mean Gaussian error with covariance $\sigma_{i}^{2}$. From a statistical point of view, the estimation error is assumed to be isotropically distributed over the three spatial directions.

Let $C_{i}$ be the optical center of the camera during the $i$-th acquisition, estimated by means of the projective reconstruction.

Assuming that the distance between the optical centers of the embedded camera and the point of the device used for the georeferenced position is negligible, then $x_{i}$ and $C_{i}$ can be related as follows:

$$
x_{i} \approx T_{g} C_{i}, \text { for } i=1, \ldots, \boldsymbol{m} .
$$

When a quite large number of camera views is available and camera measurements are quite accurate, then it can be assumed that errors on the estimated optical centers $\left\{C_{i}\right\}_{i=1, \ldots, m}$ are smaller with respect to those on the device positions $\left\{x_{i}\right\}_{i=1, \ldots, m}$. In accordance with this observation, hereafter it is assumed that the errors on the estimated optical centers are negligible with respect to those on the estimated device positions. Under this assumption the matrix $T_{g}$ can be effectively estimated as follows:

$$
T_{g}=X \Sigma_{x}^{-1} C^{\top}\left(C \Sigma_{x}^{-1} C\right)^{\dagger}
$$

where $X$ and $C$ are the matrices formed by collecting the vectors $\left\{x_{i}\right\}_{i=1, \ldots, \boldsymbol{m}}$ and $\left\{C_{i}\right\}_{i=1, \ldots, \boldsymbol{m}}$, respectively. Furthermore, the matrix $\Sigma_{x}$ is the covariance matrix of the first component of the estimates $\left\{x_{i}\right\}$, i.e. if such estimates are independent then $\Sigma_{x}$ is a diagonal $\boldsymbol{m} \times \boldsymbol{m}$ matrix. If the estimation errors on the device positions have the same variance $3 \sigma^{2}$ (equally distributed over the 3 spatial directions), then $\Sigma_{x}=\operatorname{diag}\left(\sigma^{2}, \ldots, \sigma^{2}\right)$.

Since the estimation error on the device position has been assumed to be isotropic, then $\Sigma_{x}$ is the covariance matrix for the second and third component of the estimates $\left\{x_{i}\right\}$, as well.

\section{RESULTS AND CONCLUSIONS}

Thanks to use of the ISVD algorithm the proposed reconstruction procedure allows to significantly reduce the computational time required for computation $3 \mathrm{D}$ reconstructions of the scene. The system is assumed to immediately process a newly acquired image, first computing the image features, and then computing an updated scene $3 \mathrm{D}$ reconstruction. The computation of such $3 \mathrm{D}$ reconstruction is based on the use of the ISVD algorithm, which exploits the previously computed reconstruction in order to reduce its computational burden: as shown in Fig. 4, as the number of camera views increases the computational load of the ISVD algorithm quickly becomes much lower than that of the SVD.

It is worth to notice that some issues may occur when adding new points to the Tomasi and Kanade's factorization (solved by means of the ISVD as presented in subsection 3.2): for instance, the point position cannot be computed if it is not visible by some

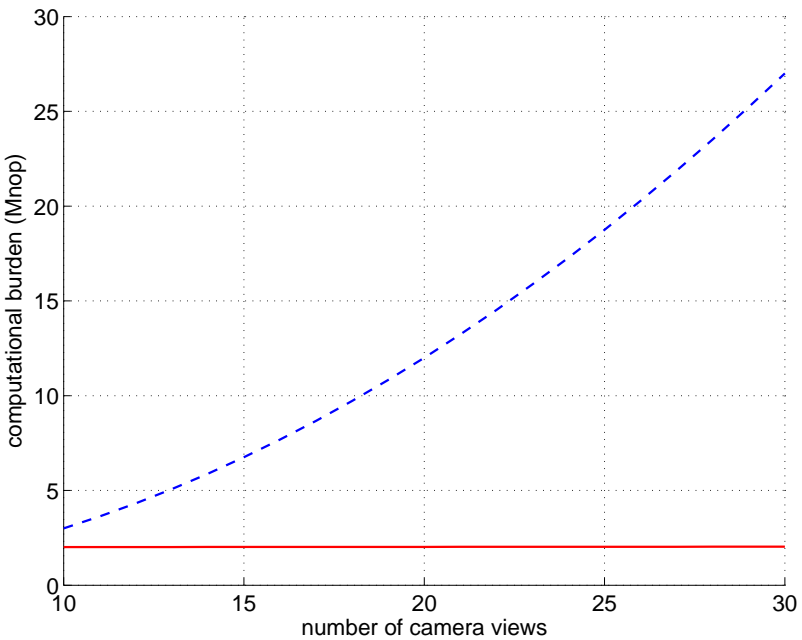

Figure 4: Comparison of the computational complexity (expressed in number of operations (nop) of one iteration of the SVD (blue dashed line) and of the ISVD (red solid line) for a matrix $W$ with size $3 \boldsymbol{m} \times n$.

cameras. Hence, a measurement related to a point have to be introduced in the computation only when it is visible by some cameras already considered in $W$. Furthermore, in certain cases (e.g. at the beginning of the algorithm) the 4-th singular value of $W$ is not so much larger than the following one: in this case neglecting the eigenvectors associated to the following singular values may lead to reconstruction errors. In order to reduce this risk, it is convenient to choose a value for $h$ larger than 4 .

Subsection 3.3 considers the problem of directly estimating the transformation that maps from the Euclidian reconstruction coordinates to the georeferenced ones. To properly validate the proposed approach a Monte Carlo simulation (with 1000 independent samples) is considered: at each iteration of the Monte Carlo simulation a new set of values for the true camera positions and for the true $T_{g}$ transformation are independently sampled. Then the estimated device and optical center positions are assumed to be affected by zero-mean random Gaussian noises with standard deviations of $0.5 \mathrm{~m}$ and $0.02 \mathrm{~m}$, respectively (accordingly with the assumption done in subsection 3.3 the variance of the error on the device positions is much larger than that of the error on the estimated optical centers). As shown in Fig. 5, the Root Mean Square Error (RMSE) of the estimated georeferenced device positions with respect to their true values decreases following the expected $1 / \sqrt{\boldsymbol{m}}$ behavior (Anderson, 2003).

In our future work, we foresee a more in depth validation of the method by considering reconstructions on different real environments, and the comparison with the results that can be obtained by means of bundle adjustment based reconstructions.

\section{REFERENCES}

Agarwal, S., Snavely, N., Seitz, S. and Szeliski, R., 2010. Bundle adjustment in the large. In: European Conference on Computer Vision, Lecture Notes in Computer Science, Vol. 6312, pp. 2942.

Anderson, T., 2003. An Introduction to Multivariate Statistical Analysis. John Wiley \& Sons Inc., New York (U.S.A.).

Azizyan, M., Constandache, I. and Choudhury, R., 2009. Surroundsense: mobile phone localization via ambience fingerprinting. In: Proceedings of the 15th annual international conference 


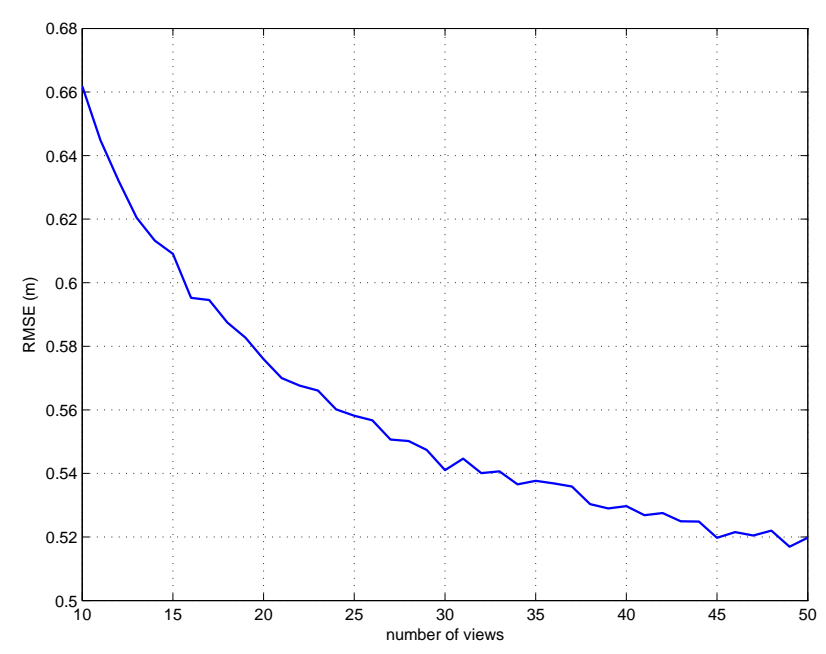

Figure 5: Estimation error of the georeferenced positions (RMSE) varying the number of camera views $\boldsymbol{m}$.

on Mobile computing and networking, MobiCom '09, pp. 261272 .

Bahl, P. and Padmanabhan, V., 2000. RADAR: An in-building RF-based user location and tracking system. In: IEEE INFOCOM, Vol. 2, pp. 775-784.

Brand, M., 2002. Incremental singular value decomposition of uncertain data with missing values. In: European Conference on Computer Vision, Lecture Notes in Computer Science, Vol. 2350, pp. 707-720.

Byröd, M. and Aström, K., 2010. Conjugate gradient bundle adjustment. In: European Conference on Computer Vision, Lecture Notes in Computer Science, Vol. 6312, pp. 114-127.

Cenedese, A., Ortolan, G. and Bertinato, M., 2010. Low-density wireless sensor networks for localization and tracking in critical environments. Vehicular Technology, IEEE Transactions on 59(6), pp. 2951-2962.

Chiuso, A., Brockett, R. and Soatto, S., 2000. Optimal structure from motion: Local ambiguities and global estimates. International Journal of Computer Vision 39(3), pp. 195-228.

El-Sheimy, N., Kai-wei, C. and Noureldin, A., 2006. The utilization of artificial neural networks for multisensor system integration in navigation and positioning instruments. Instrumentation and Measurement, IEEE Transactions on 55(5), pp. 1606-1615.

Fischler, M. and Bolles, R., 1981. Random sample consensus: A paradigm for model fitting with applications to image analysis and automated cartography. Communications of the ACM 24(6), pp. 381-395.

Foxlin, E., 2005. Pedestrian tracking with shoe-mounted inertial sensors. Computer Graphics and Applications, IEEE 25(6), pp. 38-46.

Furukawa, Y., Curless, B., Seitz, S. and Szeliski, R., 2010. Towards internet-scale multi-view stereo. In: Proceedings of the 23rd IEEE Conference on Computer Vision and Pattern Recognition (CVPR), pp. 1434-1441.

Fusiello, A., 2000. Uncalibrated euclidean reconstruction: a review. Image and Vision Computing 18(6-7), pp. 555-563.

Goesele, M., Snavely, N., Curless, B., Hoppe, H. and Seitz, S., 2007. Multi-view stereo for community photo collections. In: Proceedings of the 11th IEEE International Conference on Computer Vision (ICCV).
Guarnieri, A., Pirotti, F. and Vettore, A., 2013. Low-cost mems sensors and vision system for motion and position estimation of a scooter. Sensors 13(2), pp. 1510-1522.

Hartley, R., 1997. In defense of the eight-point algorithm. IEEE Transaction on Pattern Recognition and Machine Intelligence 19(6), pp. 580-593.

Hartley, R. and Zisserman, A., 2003. Multiple View Geometry in Computer Vision. Cambridge University Press.

Heyden, A. and Aström, K., 1996. Euclidean reconstruction from constant intrinsic parameters. In: Proceedings of the International Conference on Pattern Recognition, pp. 339-343.

Kennedy, R., Balzano, L., Wright, S. and Taylor, C., 2013. Online algorithms for factorization-based structure from motion. ArXiv p. 1309.6964 .

Longuet-Higgins, H., 1981. A computer algorithm for reconstructing a scene from two projections. Nature 293(5828), pp. 133-135.

Lukianto, C. and Sternberg, H., 2011. Stepping - smartphonebased portable pedestrian indoor navigation. Archives of photogrammetry, cartography and remote sensing 22, pp. 311-323.

Ma, Y., Soatto, S., Košecká, J. and Sastry, S., 2003. An Invitation to 3D Vision. Springer.

Masiero, A. and Cenedese, A., 2013. Affinity-based distributed algorithm for $3 \mathrm{~d}$ reconstruction in large scale visual sensor networks. In: Proceedings of the 2014 American Control Conference, ACC 2014, Portland, USA.

Masiero, A., Guarnieri, A., Vettore, A. and Pirotti, F., 2013. An indoor navigation approach for low-cost devices. In: Indoor Positioning and Indoor Navigation (IPIN 2013), Montbeliard, France.

Morel, J. and Yu, G., 2009. ASIFT: A new framework for fully affine invariant image comparison. SIAM Journal on Imaging Sciences 2(2), pp. 438-469.

Piras, M., Marucco, G. and Charqane, K., 2010. Statistical analysis of different low cost GPS receivers for indoor and outdoor positioning. In: IEEE Position Location and Navigation Symposium (PLANS), MobiSys '12, pp. 838-849.

Ruiz, A., Granja, F., Prieto Honorato, J. and Rosas, J., 2012. Accurate pedestrian indoor navigation by tightly coupling footmounted IMU and RFID measurements. Instrumentation and Measurement, IEEE Transactions on 61(1), pp. 178-189.

Snavely, N., Seitz, S. and Szeliski, R., 2008. Modeling the world from internet photo collections. International Journal of Computer Vision 80(2), pp. 189-210.

Tomasi, C. and Kanade, T., 1992. Share and motion from image streams under orthography: a factorization method. International Journal of Computer Vision 9(2), pp. 137-154.

Wang, H., Sen, S., Elgohary, A., Farid, M., Youssef, M. and Choudhury, R., 2012. No need to war-drive: unsupervised indoor localization. In: Proceedings of the 10th international conference on Mobile systems, applications, and services, MobiSys '12, pp. 197-210.

Widyawan, Pirkl, G., Munaretto, D., Fischer, C., An, C., Lukowicz, P., Klepal, M., Timm-Giel, A., Widmer, J., Pesch, D. and Gellersen, H., 2012. Virtual lifeline: Multimodal sensor data fusion for robust navigation in unknown environments. Pervasive and Mobile Computing 8(3), pp. 388-401.

Youssef, M. and Agrawala, A., 2005. The horus WLAN location determination system. In: Proceedings of the 3rd international conference on Mobile systems, applications, and services, MobiSys '05, pp. 205-218. 